\title{
Retinal Vascular Fractal Dimensions and Their Association with Macrovascular Cardiac Disease
}

\author{
Sebastian Dinesen ${ }^{a}$ Pia S. Jensen ${ }^{b, c}$ Maria Bloksgaard ${ }^{d}$ Søren Leer Blindbæk ${ }^{a}$ \\ Jo De Mey ${ }^{d, e, f} \quad$ Lars M. Rasmussen $^{b, c, g}$ Jes S. Lindholt ${ }^{c, e, g} \quad$ Jakob Grauslund ${ }^{\text {a, } g}$ \\ aDepartment of Ophthalmology, Odense University Hospital, Odense, Denmark; 'bepartment of Clinical \\ Biochemistry and Pharmacology, Odense University Hospital, Odense, Denmark; 'Centre for Individualized Medicine \\ in Arterial Diseases (CIMA), Odense University Hospital, Odense, Denmark; ${ }^{d}$ Department of Molecular Medicine, \\ University of Southern Denmark, Odense, Denmark; 'Department of Cardiac, Thoracic and Vascular Surgery, \\ Odense University Hospital, Odense, Denmark; f Department of Pharmacology and Personalized Medicine, \\ Maastricht University, Maastricht, The Netherlands; 9Department of Clinical Research, University of Southern \\ Denmark, Odense, Denmark
}

\section{Keywords}

Coronary artery bypass grafting $\cdot$ Retinal vascular geometry · Fractals · Imaging

\begin{abstract}
Introduction: As the only part of the human vasculature, the retina is available for direct, noninvasive inspection. Retinal vascular fractal dimension $\left(D_{\mathrm{F}}\right)$ is a method to measure the structure of the retinal vascular tree, with higher noninteger values between 1 and 2 representing a more complex and dense retinal vasculature. Retinal vascular structure has been associated with a variety of systemic diseases, and this study examined the association of $D_{\mathrm{F}}$ and macrovascular cardiac disease in a case-control design. Methods: Retinal fundus photos were captured with Topcon TRC-50X in 38 persons that had coronary artery bypass grafting (CABG, cases) and 37 cardiovascular healthy controls. The semiautomatic software VAMPIRE was used to measure retinal $D_{\mathrm{F}}$. Results: Patients with $C A B G$ had lower $D_{F}$ of the retinal main venular
\end{abstract}

(c) 2021 S. Karger AG, Base

www.karger.com/ore

Karger ${ }^{\prime}=$ vessels compared to the control group (1.15 vs. $1.18, p=$ 0.01 ). In a multivariable regression model adjusted for gender and age, eyes in the fourth quartile with higher $D_{F}$ were less likely to have $C A B G$ compared to patients in the first $(O R$, 7.20; 95\% confidence interval: $1.63-31.86 ; p=0.009$ ) and second (OR, 8.25; 95\% confidence interval: $1.70-40.01 ; p=$ $0.009)$ quartiles. Conclusions: This study demonstrates that lower complexity of main venular vessels associates with higher risk of having CABG. The research supports the hypothesis that the retinal vascular structure can be used to assess nonocular macrovascular disease.

(c) 2021 S. Karger AG, Basel

\section{Introduction}

The vessels of the retinal circulation are uniquely available for direct in vivo inspection. Growing evidence in various features from retinal vessel examinations, such as vessel caliber [1-6], retinal vascular fractal dimension 
$\left(D_{\mathrm{F}}\right)[7,8]$, and microvascular abnormalities $[9,10]$, suggests that retinal structural measurements can be used to detect, or even predict, cardiovascular disease.

The concept of statistical self-similarity introduced by Mandelbrot [11] in 1967 stated that a fraction of a pattern statistically can be considered the whole. In nature, this can be applied to coastal lines, snowflakes, and lightning, as well as the retinal vascular tree [12]. $D_{\mathrm{F}}$ can be measured as a noninteger between 1 and 2 . The higher the number, the more the complexity and density are represented in the retinal vascularity [13].

Macrovascular cardiac disease is traditionally described as dysfunction of the larger peripheral coronary arteries, but increasing evidence points to microvascular dysfunction as a pathogenic to macrovascular coronary artery disease $[14,15] . D_{\mathrm{F}}$ differs from other more simplified retinal vessel assessments as, for example, vessel caliber that measures only a small part of bigger retinal vessels. $D_{\mathrm{F}}$ summarizes the full pattern of the retinal circulation into a single measurable component including the smaller and bigger retinal vessels. Methods to directly measure the global coronary circulation are lacking [15], but $D_{\mathrm{F}}$ has been investigated as a surrogate measure $[7,16,17]$.

Cardiovascular disease still remains the top cause of mortality worldwide, and early risk stratification to identify and manage the diseased is essential [18]. This study aimed to investigate $D_{\mathrm{F}}$ in a population with established macrovascular cardiac disease (coronary artery bypass grafting [CABG] patients) compared to cardiovascular healthy controls.

\section{Materials and Methods}

Study examinations were performed from March 2018 until ultimo December 2018 at Odense University Hospital (OUH), Odense, Denmark. The study population consisted of a group suffering from macrovascular cardiac disease (43 cases) and a cardiovascular healthy group (42 controls).

Cases were recruited at the Department of Cardiac, Thoracic and Vascular Surgery, OUH, where they underwent CABG. Recruitment took place 4 days after surgery and the clinical examinations when they had pace wires and heart monitoring scope discontinued 4 to 6 days after surgery. Controls were recruited at the Department of Ophthalmology, OUH, on the day after they underwent cataract surgery.

Exclusion criteria included mental inability, age under 18, retinal disease (i.e., diabetic retinopathy or hypertensive retinopathy), and the need for resurgery applicable for both groups. Cases had to be CABG patients and were excluded if they had CABG combined with cardiac valve surgery. Controls could not have any history or presence of cardiac disease. Controls were not excluded if they had presence of cardiac risk factors such as hypertension, hy-

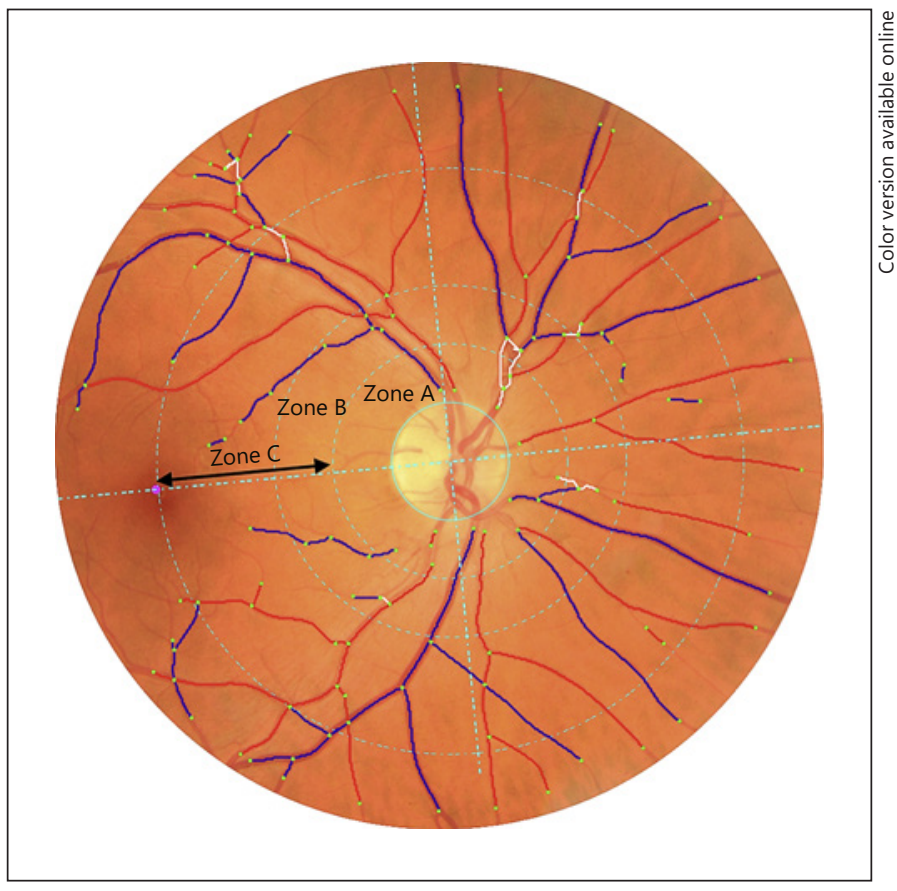

Fig. 1. A disc-centered fundus photography processed for analysis of fractal dimension by using the VAMPIRE software (Vessel Assessment and Measurement Platform for Images of the REtina; The Vampire Group, Edinburgh, United Kingdom). The blue and red vessels represent venules and arterioles, respectively. The coordinate system surrounding the optic disc is marked with zones $\mathrm{A}, \mathrm{B}$, and $\mathrm{C}$ located $0.0-0.5,0.5-1.0$, and $0.5-2.0$ disc diameters from the optic disc center.

percholesterolemia, diabetes, and history of smoking, or if they were treated with anticoagulants from noncardiac causes.

Clinical characteristics were gathered from a full medical interview in addition to information from the patient records. After 10 minutes of rest, blood pressure was measured and standardized as a mean of 3 measurements on the right upper arm (Omron M6 AC, Kyoto, Japan). Blood samples were collected for analysis including cholesterol profile, triglycerides, and hemoglobin A1c. The Topcon TRC-50X (Topcon, Tokyo, Japan) was used to capture a $45^{\circ}$ optic disc-centered fundus photography from the right eye (or left eye, if the right was not eligible for inclusion) after mydriasis was achieved with tropicamide $1 \%$ and phenylephrine $10 \%$.

The VAMPIRE (Vessel Assessment and Measurement Platform for Images of the REtina; The Vampire Group, Edinburgh, United Kingdom) software was used for $D_{\mathrm{F}}$ analysis based on a prespecified protocol. The grader was not blinded to the group allocation of the individual participant. The method for the semiautomatic software is outlined in detail elsewhere $[19,20]$ and will only be briefly explained here.

Retinal landmarks were automatically detected, including the center and outer diameter of the optic disc and the macula center. This enabled the establishment of a coordinate system with circular zones A ( $0-0.5$ disc diameters from the optic disc center), B (between 0.5 and 1.0), and $C$ (between 0.5 and 2.0) around the optic disc (Fig. 1). Vessels were automatically traced as arterioles or venules, 
Fig. 2. A retinal image (a) and its corresponding skeletonized image (b) illustrating the refined line tracing of the retinal vasculature.

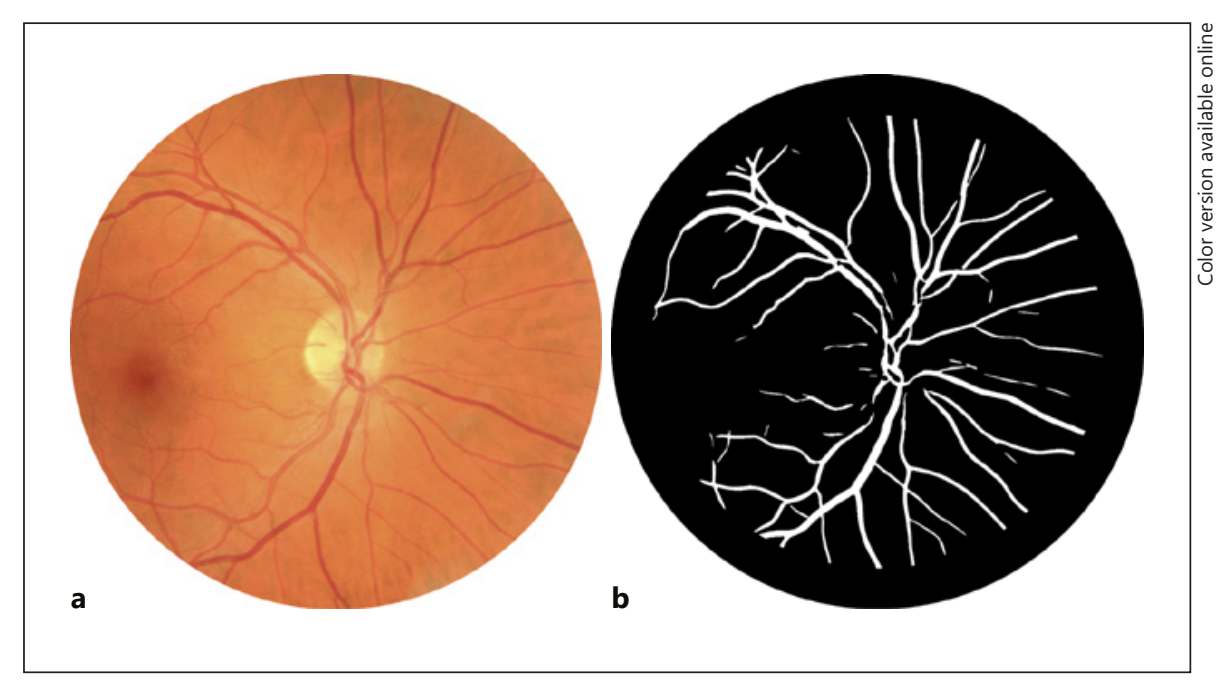

ranging from zone $\mathrm{A}$ and peripheral to zone $\mathrm{C}$. This creates a skeletonized pattern (Fig. 2b) of the entire retinal circulation (Fig. 2a). The automatic localization of the optic disc, macula, and vessels was not always on point, and manual corrections were made when needed. In some cases, traced vessels were erased when it was unclear which vessel type was represented and when VAMPIRE misinterpreted an artefact or the underlying choroid layer as a retinal vessel.

The software ran the final analysis by the box-counting method $[19,21,22]$ developed for self-similar patterns that are not completely self-similar. Those sequences of the arterioles and venules coursing through zone $\mathrm{C}$ are included to calculate $D_{\mathrm{F}}$. Outcomes were arteriolar, venular, and total $D_{\mathrm{F}}$ from all vessels traced in zone $\mathrm{C}$ as well as $D_{\mathrm{F}}$ from the main vessels alone. The method is validated with a high intergrader reproducibility [19].

Continuous data are presented as medians and categorical data as percentages. The 0.05 level was used to test for all statistical differences using Stata Intercooled 16 (StataCorp, College Station, TX, USA).

Differences between the 2 groups were tested by the MannWhitney $U$ test used for continuous data and the Pearson $\chi^{2}$ used for categorical data. A multivariable logistic regression model with adjustment for gender and age was used to display the odds ratio of macrovascular cardiovascular disease in relation to main venular fractal dimension divided into 4 quartiles.

\section{Results}

The exclusion process led to 10 exclusions leaving 75 participants for further analysis (37 controls and 38 cases). Eight were excluded due to poor image quality, one due to former central retinal vein occlusion, and one because of former cardiac disease (control group).

Differences between the 2 groups are shown in Table 1 . The case group consisted of more men (76.3 vs. $51.4 \%, p=0.02)$, had a higher BMI (28.0 vs. $25.5, p=0.02)$, and were more likely to have diabetes mellitus (34.2 vs.
$8.1 \%, p=0.01$ ), but had a lower systolic (138 vs. $143 \mathrm{~mm}$ $\mathrm{Hg}, p=0.01$ ) and diastolic (70 vs. $90 \mathrm{~mm} \mathrm{Hg}, p=<0.001$ ) blood pressure. Cases were more commonly medicated with antihypertensives ( 89.5 vs. $51.4 \%, p=<0.001)$, statins (94.7 vs. $29.7 \%, p=<0.001$ ), and anticoagulants (100 vs. $21.6 \% p=<0.001)$.

We found no differences between the 2 groups in age, smoking history, HDL-cholesterol, triglycerides, and HbAlc. As compared to eyes of control patients, eyes of cases had lower retinal fractal dimension regarding the main venules ( 1.15 vs. $1.18, p=0.01)$, but did not differ according to other retinal fractal measurements (Table 1).

Table 2 displays the odds ratio of having CABG in relation to the main venular fractals. The VAMPIRE software was unable to calculate the main venular $D_{\mathrm{F}}$ in one of our cases. In the multivariable analysis adjusted for gender and age, eyes of patients with the highest values in $D_{\mathrm{F}}$ (fourth quartile) were less likely to have been operated for CABG as compared to the eye of patients in the first (OR, 7.20; 95\% confidence interval: $1.63-31.86 ; p=0.009$ ) and second (OR, 8.25; 95\% confidence interval: 1.70$40.01 ; p=0.009)$ quartiles. In fact, the risk of CABG increased with $83 \%$ for each standard deviation decrease in main venular fractal dimension (OR, 1.83; 95\% confidence interval: $1.07-3.13 ; p=0.03)$.

\section{Discussion}

The present study demonstrated an association between $C A B G$ and retinal main venular $D_{\mathrm{F}}$ when compared to cardiovascular healthy controls. In particular, eyes of patients from the 2 lowest quartiles compared to 
Table 1. Clinical and demographic characteristics of eyes of patients operated with CABG (cases) as compared to eyes from cardiovascular healthy controls

\begin{tabular}{|c|c|c|c|}
\hline & $\begin{array}{l}\text { Cases } \\
(n=38)\end{array}$ & $\begin{array}{l}\text { Controls } \\
(n=37)\end{array}$ & $p$ value \\
\hline Gender, men, \% & 76.3 & 51.4 & 0.02 \\
\hline Age, years & 70.5 & 72.0 & 0.28 \\
\hline Body mass index, $\mathrm{kg} / \mathrm{m}^{2}$ & 28.0 & 25.5 & 0.02 \\
\hline Systolic blood pressure, $\mathrm{mm} \mathrm{Hg}$ & 138 & 143 & 0.01 \\
\hline Diastolic blood pressure, $\mathrm{mm} \mathrm{Hg}$ & 70 & 90 & $<0.001$ \\
\hline History of smoking, $\%$ & 71.4 & 78.3 & 0.48 \\
\hline Diabetes mellitus, \% & 34.2 & 8.1 & 0.01 \\
\hline \multicolumn{4}{|l|}{ Medication, $\%$} \\
\hline Antihypertensive & 89.5 & 51.4 & $<0.001$ \\
\hline Cholesterol-lowering & 94.7 & 29.7 & $<0.001$ \\
\hline Anti-coagulation & 100 & 21.6 & $<0.001$ \\
\hline \multicolumn{4}{|l|}{ Plasma levels } \\
\hline Total-cholesterol, $\mathrm{mmol} / \mathrm{L}$ & 3.4 & 5.6 & 0.01 \\
\hline HDL-cholesterol, mmol/L & 1.3 & 1.5 & 0.18 \\
\hline LDL-cholesterol, mmol/L & 1.7 & 3.4 & 0.02 \\
\hline Triglycerides, $\mathrm{mmol} / \mathrm{L}$ & 1.4 & 1.4 & 0.37 \\
\hline $\mathrm{HbAlc}, \mathrm{mmol} / \mathrm{mol}$ & 37.0 & 37.5 & 0.85 \\
\hline \multicolumn{4}{|l|}{ Vessel geometry } \\
\hline Arteriolar fractal dimension & $1.24 \pm 0.05$ & $1.23 \pm 0.05$ & 0.45 \\
\hline Venular fractal dimension & $1.21 \pm 0.05$ & $1.22 \pm 0.04$ & 0.44 \\
\hline Total fractal dimension & $1.39 \pm 0.04$ & $1.38 \pm 0.03$ & 0.47 \\
\hline Arteriolar fractal dimension main vessels & $1.15 \pm 0.06$ & $1.15 \pm 0.08$ & 0.90 \\
\hline Venular fractal dimension main vessels & $1.15 \pm 0.06$ & $1.18 \pm 0.05$ & $0.01^{*}$ \\
\hline Total fractal dimension main vessels & $1.32 \pm 0.06$ & $1.33 \pm 0.07$ & 0.35 \\
\hline
\end{tabular}

Data are presented as medians. Tested for differences between cases and controls. $p$ values $<0.05$ are regarded statistically significant. History of smoking = former or current; antihypertensive medication = all kinds of antihypertensive drugs; CABG, coronary artery bypass grafting; HDL-cholesterol, high-density lipoprotein-cholesterol; LDL-cholesterol, low-density lipoprotein-cholesterol; HbA1c, glycosylated hemoglobin. * Statistically significant.

Table 2. Association of retinal main venular fractal dimensions and coronary artery bypass surgery in a model adjusted for age and gender

\begin{tabular}{lll}
\hline $\begin{array}{l}\text { Retinal main venular } \\
\text { fractal dimension }\end{array}$ & $N$ & Cardiovascular disease \\
\hline Per SD decrease, 0.0555 & & $1.83(1.07-3.13)^{*}$ \\
First quartile, $1.017-1.123$ & 20 & $7.20(1.63-31.86)^{*}$ \\
Second quartile, $1.124-1.162$ & 18 & $8.25(1.70-40.01)^{*}$ \\
Third quartile, $1.164-1.200$ & 18 & $1.79(0.39-8.15)$ \\
Fourth quartile, $1.201-1.324$ & 18 & 1 (reference) \\
\hline
\end{tabular}

$\mathrm{SD}$, standard deviation. 4th quartile functions as reference for quartiles $1-3$. Results are presented as odds ratios with $95 \%$ confidence intervals. ${ }^{*}$ Statistically significant. patients from the top quartile had a 7.2 and 8.2 times higher risk of having CABG, respectively.

While the correlation between retinal vascular structure and CABG has not been addressed before, other researchers studied $D_{\mathrm{F}}$ and its association with cardiovascular mortality as well as risk factors $[7,8]$. Lower and greater $D_{\mathrm{F}}$ were associated with higher risk of 14-year cardiovascular disease mortality in a prospective cohort study by Liew et al. [7]. Patients with $D_{\mathrm{F}}$ in the first quartile had a 44\% (OR, 1.44; 95\% CI: 1.13-1.83) increased risk of cardiovascular mortality, while patients in the fourth quartile had a $51 \%$ (OR, 1.52; 95\% CI: 1.14-1.98) increased risk compared to patients in the second and third quartiles. Low $D_{\mathrm{F}}$ and increased risk of cardiovascular disease support the findings in our study, but cannot be directly transferred, since we only found differences for the retinal main venular $D_{\mathrm{F}}$. 
Cheung et al. [8] investigated the association between $D_{\mathrm{F}}$ and cardiovascular risk factors in a cross-sectional design showing that higher mean arterial blood pressure (coefficient $-0.085 ; p<0.001$ ) and older age (coefficient $-0.311 ; p<0.001)$ were associated with lower $D_{\mathrm{F}}$. Liew et al. [23] made a similar observation with lower $D_{\mathrm{F}}$ being associated with elevated systolic blood pressure (coefficient $-0.29 ; p<0.0001$ ).

According to the principle of minimum work stated by Murray [24], the human circulation has an architectural optimum securing the delivery of oxygen to the tissues. $D_{\mathrm{F}}$ is a potential method to evaluate deviations from this optimal architectural structure providing new insights to circulatory diseases.

The pathophysiological mechanisms leading to suboptimal $D_{\mathrm{F}}$ are not well understood. However, it is well known that cardiovascular risk factors such as hypertension, elevated cholesterol levels, and diabetes all dispose to atherosclerosis and microvascular alterations [25]. Antihypertensives, anticoagulants, and cholesterol-lowering drugs were all represented with statistical significance in the case group (Table 1). This indicates that cardiovascular risk factors have been present disposing to alterations in the retinal vascular complexity perhaps resulting in lower $D_{\mathrm{F}}$. Table 1 also displays a higher representation of diabetes among the cases. Diabetic retinopathy and lower $D_{\mathrm{F}}$ were associated in the study by Grauslund et al. [26], and on the basis of this, diabetes is a potential influencing parameter in the present study.

However, these are all speculations, and the underlying mechanisms regarding lower $D_{\mathrm{F}}$ are poorly understood, and more research is needed. There is a lot of potential that retinal vascular $D_{\mathrm{F}}$, as a noninvasive procedure, can be implemented to help clinicians evaluate and maybe even predict the risk of cardiovascular disease based on the alterations in the geometric complexity of the retinal vasculature. This involves a well-validated software application such as VAMPIRE, since the retinal vascular $D_{\mathrm{F}}$ is difficult to evaluate with the naked eye as a clinician. The difference in $D_{\mathrm{F}}$ between the lowest and highest quartiles is in particular what is clinically interesting since patients in the lowest quartile had an increased risk of having CABG. Future research should include functional measure of retinal ischemia in association with $D_{\mathrm{F}}$ to better understand the retinal pathophysiology and a larger population to better evaluate the prognostic value of $D_{\mathrm{F}}$ for CABG. Secondly, it would be interesting to investigate whether the retinal microcirculation correlates with the cardiovascular microcirculation.

Retinal Vascular Fractals and CABG
Our study contains some limitations that should be mentioned. First of all, the cross-sectional design is limiting, as it does not allow elucidating causal relationships. Secondly, given the age of the study population, it was not possible to include controls without any cardiovascular risk factors, even though these were statistically significantly lower than the cases. The results are potentially influenced by the fact that cardiovascular risk factors were to some extent also present in the control group, even though these patients did not have manifest cardiovascular disease.

Refractive errors affect retinal measures in general, and Cheung et al. [8] observed an association between myopic refraction and lower retinal vascular $D_{\mathrm{F}}$. We were not able to correct for myopia or other refractive deviations, which potentially is reflected in our overall findings. Further, we did not manage to match the groups on cardiovascular parameters, but, on the other hand, it is considered a strength that the groups are matched in age, due to research suggesting that $D_{\mathrm{F}}$ decreases with age $[8$, 27].

\section{Conclusion}

In summary, this study demonstrated that retinal vascular fractals, a method to assess the complexity of the retinal vascularity, associate with macrovascular cardiac disease after adjusting for gender and age. Prospective and functional studies are needed to clarify the pathophysiological mechanisms fundamental to understand the full picture. Nonetheless, we were able to shed light on the promising potential the retinal circulation has when it comes to assessing not only ocular diseases but also extraocular diseases.

\section{Statement of Ethics}

The project was approved by the regional ethics committee (project-ID: S-20170205) and the Danish Data Agency (Journal No. 18/29227). It was performed according to good clinical practice and the Declaration of Helsinki. The participants all gave their written informed consent on a voluntary and informed basis.

\section{Conflict of Interest Statement}

There are no conflicts of interest. 


\section{Funding Sources}

The Velux Foundation supported this work.

\section{Author Contributions}

All authors listed have contributed to the design and manuscript. The first author did the inclusion of patients and the analysis of data.

\section{References}

1 McGeechan K, Liew G, Macaskill P, Irwig L, Klein R, Klein BE, et al. Meta-analysis: retinal vessel caliber and risk for coronary heart disease. Ann Intern Med. 2009;151(6):404-13.

2 Seidelmann SB, Claggett B, Bravo PE, Gupta A, Farhad H, Klein BE, et al. Retinal vessel calibers in predicting long-term cardiovascular outcomes: the atherosclerosis risk in communities study. Circulation. 2016;134(18): 1328-38.

3 Sun C, Liew G, Wang JJ, Mitchell P, Saw SM, Aung T, et al. Retinal vascular caliber, blood pressure, and cardiovascular risk factors in an Asian population: the Singapore Malay Eye Study. Invest Ophthalmol Vis Sci. 2008;49(5): 1784-90.

4 Wang JJ, Liew G, Wong TY, Smith W, Klein $\mathrm{R}$, Leeder SR, et al. Retinal vascular calibre and the risk of coronary heart disease-related death. Heart. 2006;92(11):1583-7.

5 Wong TY, Islam FM, Klein R, Klein BE, Cotch MF, Castro C, et al. Retinal vascular caliber, cardiovascular risk factors, and inflammation: the multi-ethnic study of atherosclerosis (MESA). Invest Ophthalmol Vis Sci. 2006; 47(6):2341-50.

6 Wong TY, Kamineni A, Klein R, Sharrett AR, Klein BE, Siscovick DS, et al. Quantitative retinal venular caliber and risk of cardiovascular disease in older persons: the cardiovascular health study. Arch Intern Med. 2006;166(21): 2388-94.

7 Liew G, Mitchell P, Rochtchina E, Wong TY, Hsu W, Lee ML, et al. Fractal analysis of retinal microvasculature and coronary heart disease mortality. Eur Heart J. 2011;32(4):422-9.

8 Cheung CY, Thomas GN, Tay W, Ikram MK, Hsu W, Lee ML, et al. Retinal vascular fractal dimension and its relationship with cardiovascular and ocular risk factors. Am J Ophthalmol. 2012;154(4):663-74.e1.
9 Wong TY, Klein R, Nieto FJ, Klein BE, Sharrett AR, Meuer SM, et al. Retinal microvascular abnormalities and 10-year cardiovascular mortality: a population-based case-control study. Ophthalmology. 2003;110(5):933-40.

10 Witt N, Wong TY, Hughes AD, Chaturvedi N, Klein BE, Evans R, et al. Abnormalities of retinal microvascular structure and risk of mortality from ischemic heart disease and stroke. Hypertension. 2006;47(5):975-81.

11 Mandelbrot B. How long is the coast of britain? Statistical self-similarity and fractional dimension. Science. 1967;156(3775):636-8.

12 Masters BR. Fractal analysis of the vascular tree in the human retina. Annu Rev Biomed Eng. 2004;6:427-52.

13 Ab Hamid F, Che Azemin MZ, Salam A, Aminuddin A, Mohd Daud N, Zahari I. Retinal vasculature fractal dimension measures vessel density. Curr Eye Res. 2016;41(6):823-31.

14 Camici PG, Crea F. Coronary microvascular dysfunction. N Engl J Med. 2007;356(8):83040.

15 Crea F, Camici PG, Bairey Merz CN. Coronary microvascular dysfunction: an update. Eur Heart J. 2014;35(17):1101-11.

16 Liew G, Wang JJ, Mitchell P, Wong TY. Retinal vascular imaging: a new tool in microvascular disease research. Circ Cardiovasc Imaging. 2008;1(2):156-61

17 Patton N, Aslam TM, MacGillivray T, Deary IJ, Dhillon B, Eikelboom RH, et al. Retinal image analysis: concepts, applications and potential. Prog Retin Eye Res. 2006;25(1):99127.

18 Collaborators GBDCoD. Global, regional, and national age-sex specific mortality for 264 causes of death, 1980-2016: a systematic analysis for the Global Burden of Disease Study 2016. Lancet. 2017;390(10100):1151-210.
19 Perez-Rovira A, MacGillivray T, Trucco E, Chin KS, Zutis K, Lupascu C, et al. VAMPIRE: vessel assessment and measurement platform for images of the REtina. Conf Proc IEEE Eng Med Biol Soc. 2011;2011:3391-4.

20 Emanuele Trucco AG, Ballerini L, Relan D, Cavinato A, MacGillivray T. Morphometric measurements of the retinal vasculature in fundus images with VAMPIRE Biomedical image understanding: methods and applications. Hoboken, NJ: John Wiley \& Sons; 2015. p. 91-111.

21 Macgillivray TJ, Patton N, Doubal FN, Graham C, Wardlaw JM. Fractal analysis of the retinal vascular network in fundus images. Conf Proc IEEE Eng Med Biol Soc. 2007;2007: 6456-9.

22 Stosic T, Stosic BD. Multifractal analysis of human retinal vessels. IEEE Trans. Med. Imaging. 2006;25(8):1101-7.

23 Liew G, Wang JJ, Cheung N, Zhang YP, Hsu $\mathrm{W}$, Lee $\mathrm{ML}$, et al. The retinal vasculature as a fractal: methodology, reliability, and relationship to blood pressure. Ophthalmology. 2008; 115(11):1951-6.

24 Murray CD. The physiological principle of minimum work: I. The vascular system and the cost of blood volume. Proc Natl Acad Sci U S A. 1926;12(3):207-14.

25 Farah C, Michel LYM, Balligand JL. Nitric oxide signalling in cardiovascular health and disease. Nat Rev Cardiol. 2018;15(5):292-316.

26 Grauslund J, Green A, Kawasaki R, Hodgson L, Sjølie AK, Wong TY. Retinal vascular fractals and microvascular and macrovascular complications in type 1 diabetes. Ophthalmology. 2010;117(7):1400-5.

27 Doubal FN, MacGillivray TJ, Patton N, Dhillon B, Dennis MS, Wardlaw JM. Fractal analysis of retinal vessels suggests that a distinct vasculopathy causes lacunar stroke. Neurology. 2010;74(14):1102-7. 